\title{
Results of a questionnaire regarding criteria for adequacy of endometrial biopsies
}

\section{Phillips, W G McCluggage}

\begin{abstract}
Aims: Pathologists are faced with increasing numbers of endometrial biopsies containing scant tissue. Anecdotal evidence points to significant variation among pathologists regarding criteria used to assess adequacy, and no standard recommendations exist. An initial audit showing variation in endometrial biopsy adequacy reporting prompted this assessment of the criteria used by specialist gynaecological pathologists for the classification of adequacy.

Methods: A questionnaire regarding criteria used for endometrial biopsy assessment adequacy was sent to members of the British Association of Gynaecological Pathologists and the National Gynaecological Pathology External Quality Assessment Scheme (UK). One hundred and thirty questionnaires were distributed and 61 pathologists responded.

Results: The responses showed great variation in criteria used to classify endometrial biopsies as adequate. Most respondents felt it would be useful if criteria were proposed to aid this assessment.

Conclusions: Wide variation exists among specialist gynaecological pathologists regarding what constitutes an adequate endometrial biopsy. The gynaecologist should interpret the biopsy report in the light of clinical, radiological, and hysteroscopic features. The presence of scanty tissue in postmenopausal women with a thin endometrium and no focal lesion is expected, and is not a reason for repeat biopsy. Pathologists should exercise caution before classifying endometrial biopsies as inadequate, because this may have medicolegal and management implications.
\end{abstract}

W ith the increasing trend to perform outpatient endometrial pipelle biopsies rather than formal curettage, pathologists are dealing with increasing numbers of endometrial specimens in which there is scanty, or even no, endometrial tissue. In such instances, it is controversial as to what constitutes an adequate or inadequate specimen. The designation of a biopsy as inadequate may be of importance because this can have management and medicolegal implications. For example, some clinicians routinely perform a repeat biopsy when an earlier sample has been reported as inadequate, whereas others do not.

"There are currently no standard criteria to determine what represents an adequate endometrial biopsy, and there is anecdotal evidence that criteria used by pathologists are varied and inconsistent"

A biopsy reported as inadequate may suggest to some that the clinician is at fault, or has not undertaken the biopsy procedure correctly. Although this may be the case in some instances, in most cases it is not. In published studies, inadequate rates of outpatient endometrial biopsies range from $4.8 \%$ to $33 \%{ }^{1-5}$ but in most of these studies criteria for adequacy are not stated. One study defines an adequate sample as one or more pieces of endometrium large enough to determine the gland to stroma ratio and endometrial morphological features, and an inadequate sample as consisting only of blood or cervical mucous with fragments of benign endocervix, or a large amount of blood with only small fragments of endometrial glands and stroma. ${ }^{6}$ There are currently no standard criteria to determine what represents an adequate endometrial biopsy, and there is anecdotal evidence that criteria used by pathologists are varied and inconsistent. For example, in a recent audit performed in our department, only 53\% of endometrial biopsies reported as inadequate by general histopathologists (without an interest in gynaecological pathology) would have been designated as such by one of the authors of our present study (WGM, a specialist gynaecological pathologist). The results of this audit are presented here. In addition, we distributed a questionnaire among a group of gynaecological pathologists in the UK with a view to ascertaining criteria used for the assessment of adequacy of an endometrial biopsy.

\section{MATERIALS AND METHODS}

An audit was carried out in the department of pathology, Royal Group of Hospitals Trust, Belfast, UK regarding endometrial biopsies reported as inadequate or insufficient. A SNOMED computer search was performed to ascertain the total number of endometrial biopsies reported and to identify those reported as inadequate over a 12 month period (1 September 2002 to 31 August 2003). This was before the introduction of a subspecialist reporting system in the department. Cases reported as inadequate by non-specialist pathologists were reviewed by WGM to see whether he agreed with this assessment. The criteria used by WGM regarding adequacy of endometrial biopsies are: the term inadequate or insufficient is used only when no endometrial tissue is represented, whereas the term unassessable is used when a minimal amount of endometrium is present such that the endometrium cannot be typed. When the endometrium can be typed this is done regardless of how little tissue is represented. It is recognised that these criteria are in themselves subjective and that there is no evidence base for these.

A questionnaire (table 1) was formulated to look at criteria used by gynaecological pathologists for the assessment of adequacy of endometrial biopsies. This was sent to all members of the British Association of Gynaecological Pathologists and participants in the National Gynaecological Pathology External Quality Assessment Scheme in the UK, along with a stamped addressed envelope for returning the questionnaire. One hundred and thirty questionnaires were distributed and 61 (46.9\%) were completed and returned. The results were analysed by the authors of the study.

\section{RESULTS}

During the period of the audit there were 1280 endometrial biopsies, of which 99 were reported as inadequate or 
Table 1 Questionnaire regarding criteria used for assessment of adequacy of endometrial biopsies with opinions of respondents

\begin{tabular}{|c|c|c|c|}
\hline Question & Yes & No & Not answered \\
\hline $\begin{array}{l}\text { Does your reporting depend on whether the specimen is a pipelle biopsy or } \\
\text { curette? }\end{array}$ & $29 / 61(47.5 \%)$ & $30 / 61(49.2 \%)$ & $2 / 61(3.3 \%)$ \\
\hline $\begin{array}{l}\text { Does your reporting depend on whether the patient is premenopausal or } \\
\text { postmenopausal? }\end{array}$ & $40 / 61(65.6 \%)$ & $18 / 61(29.5 \%)$ & $3 / 61(4.9 \%)$ \\
\hline $\begin{array}{l}\text { Does your report require correlation with parameters such as ultrasonic or } \\
\text { hysteroscopic features? }\end{array}$ & $35 / 61(57.4 \%)$ & $21 / 61$ (34.4\%) & $5 / 61(8.2 \%)$ \\
\hline \multicolumn{4}{|l|}{$\begin{array}{l}\text { What do you require to classity an endometrial biopsy as adequate (only } \\
\text { answer one of these questions)? }\end{array}$} \\
\hline Any endometrial tissue no matter how scant & $6 / 61(9.8 \%)$ & & \\
\hline A certain number of superficial endometrial glands & $6 / 61(9.8 \%)$ & & \\
\hline Both endometrial glands and stroma, no matter how little & $12 / 61(19.7 \%)$ & & \\
\hline A specific number of endometrial glands and associated stroma in intact & $5 / 6](8.2 \%)$ & & \\
\hline $\begin{array}{l}\text { A specitic number of endometrial glands and associated stroma in infact } \\
\text { organised tissue }\end{array}$ & $3 / 61(8.2 \%)$ & & \\
\hline Other: please specify & $9 / 61(14.8 \%)$ & & \\
\hline \multicolumn{4}{|l|}{$\begin{array}{l}\text { Would you classify an endometrial biopsy as inadequate if the following } \\
\text { applied? }\end{array}$} \\
\hline No endometrial tissue is present in a premenopausal woman & $57 / 61(93.4 \%)$ & $2 / 61(3.3 \%)$ & $2 / 61(3.3 \%)$ \\
\hline No endometrial tissue is present in a postmenopausal woman & $56 / 61(91.8 \%)$ & $4 / 61(6.6 \%)$ & $1 / 61(1.6 \%)$ \\
\hline No endometrial tissue is present in a postmenopausal woman and hystero- & $46 / 61(75.4 \%)$ & $14 / 61(23.0 \%)$ & $1 / 61(1.6 \%)$ \\
\hline scopy and ultrasonography show atrophic endometrium & & & \\
\hline A few superficial glands without stroma are present in a premenopausal & $47 / 61(77.0 \%)$ & $10 / 61(16.4 \%)$ & $4 / 61(6.6 \%)$ \\
\hline \multicolumn{4}{|l|}{ woman } \\
\hline A few superficial glands without stroma are present in a postmenopausal woman & $29 / 61(47.5 \%)$ & $27 / 61(44.3 \%)$ & $5 / 61(8.2 \%)$ \\
\hline Stroma and no glands are present in a premenopausal & $49 / 61(80.3 \%)$ & $7 / 61(11.5 \%)$ & $5 / 61(8.2 \%)$ \\
\hline \multicolumn{4}{|l|}{ woman } \\
\hline Stroma and no glands are present in a postmenopausal woman & $45 / 61(73.8 \%)$ & $11 / 61(18.0 \%)$ & $5 / 61(8.2 \%)$ \\
\hline Less than 5 glands with a little stroma are present & $21 / 61(34.4 \%)$ & $30 / 61(49.2 \%)$ & $10 / 61(16.4 \%)$ \\
\hline Do you think suggested criteria for adequacy/inadequacy would be useful? & $54 / 61(88.5 \%)$ & $5 / 61(8.2 \%)$ & $2 / 61(3.3 \%)$ \\
\hline Do you think the term "unassessable" would be useful in some cases? & $26 / 61(42.6 \%)$ & $28 / 61(45.9 \%)$ & $7 / 61(11.5 \%)$ \\
\hline Do you use any term other than inadequate or insufficient? & $27 / 61(44.3 \%)$ & $11 / 61(18.0 \%)$ & $23 / 61(37.7 \%)$ \\
\hline
\end{tabular}

insufficient. Sixty two of these were reported by general pathologists with no specific interest in gynaecological pathology. On review of these 62 biopsies by WGM, 33 were considered inadequate, 20 were considered unassessable, and nine were considered cyclical or atrophic.

Sixty one $(46.9 \%)$ pathologists responded to the questionnaire. Table 1 shows the responses. In the section regarding other criteria used to classify an endometrial biopsy as adequate, the suggestions varied widely. For example, some required a specific number of endometrial glands (range, 5-20), whereas others required the presence of endometrial glands and intact stroma. Some respondents felt that the criteria for adequacy depended on whether the biopsy was a pipelle sample or a curette, whereas others used different criteria with regard to premenopausal and postmenopausal women. Some felt that the endometrial appearance, based on hysteroscopic and ultrasonic examination, influenced reporting, whereas others felt that it did not.

- Insufficient to be considered representative when ultrasound or hysteroscopy suggests thickened endometrium

- Insufficient for reliable assessment

- Non-diagnostic

- Suboptimal

- Scanty

- Insufficient for assessment of endometrial morphology

- Insufficient to exclude the clinical diagnosis

- No malignancy/significant features seen in a scanty specimen

- No formed endometrium

- Features may not be representative

- Tissue too scant for complete assessment

Figure 1 Examples of suggested alternative terminologies to inadequate or insufficient.
Many respondents commented on the subjectivity involved in the reporting process, and the requirement for a "common sense" and "evidence based" approach. Most respondents felt it would be useful if criteria were suggested for assessing the adequacy of an endometrial biopsy.

In the section regarding alternative terminologies to inadequate or insufficient, there was a variety of suggestions (examples shown in fig 1). Some respondents felt that using terms other then inadequate or insufficient is confusing for clinicians, whereas others stated that local clinicians had requested that these terms should be avoided because of management implications.

\section{DISCUSSION}

The impetus for the audit and subsequent questionnaire was the perception that among both general histopathologists and specialist gynaecological pathologists there is considerable variation in the criteria used to categorise an endometrial biopsy as adequate or inadequate. The number of scanty endometrial biopsies has greatly increased since the widespread introduction of outpatient pipelle biopsies for the investigation of abnormal uterine bleeding. Before this, most endometrial biopsy specimens were obtained by dilatation and curettage, a procedure that requires an anaesthetic, with resultant time and cost implications. There is also a perception that gynaecologists respond in various ways to a biopsy report of an inadequate endometrial sample (numerous personal communications). Some gynaecologists routinely rebiopsy in this situation (with resultant workload implications for histopathology laboratories), whereas others do not unless there is a clinical suspicion of hyperplasia or malignancy, or when transvaginal ultrasound or hysteroscopic examination show a thickened endometrium or a focal lesion. $^{78}$ In this regard, it is worth noting that many gynaecologists routinely perform transvaginal ultrasound or 


\section{Take home messages}

- The results of a questionnaire regarding criteria for adequacy of endometrial biopsies revealed wide variation among specialist gynaecological pathologists regarding what constitutes an adequate endometrial biopsy

- Most respondents felt it would be useful if criteria were proposed to aid the assessment of endometrial biopsy adequacy

- We suggest that the gynaecologist should interpret the biopsy report in the light of the clinical, radiological, and hysteroscopic features

- The presence of scanty tissue in an endometrial biopsy in a postmenopausal woman with a thin endometrium and no focal lesion is to be expected, and is not a reason for a repeat endometrial biopsy

- Pathologists should exercise caution before classifying an endometrial biopsy as inadequate because this may have medicolegal and management implications

hysteroscopy in conjunction with an outpatient endometrial biopsy. It is also worth noting that studies have shown that with an atrophic endometrium and no focal lesion, minimal tissue is the norm with a pipelle biopsy, and there is little chance of missing important pathology. ' It has been suggested that in a patient with postmenopausal bleeding and an endometrial thickness less than $6 \mathrm{~mm}$, biopsy is unnecessary. ${ }^{8}$ In contrast, another study suggests that in women with postmenopausal bleeding, an inadequate sample is an indication for further investigations. ${ }^{6}$ However, this approach is fraught with difficulties because, as discussed and as shown by our study, criteria used by pathologists to categorise an endometrial biopsy as inadequate vary widely.

"We suggest that caution should be exercised before categorising an endometrial biopsy as inadequate or insufficient"

Almost half of respondents to the questionnaire thought that the assessment of adequacy depends on whether the specimen comprises a pipelle biopsy or a curette. However, this is not always stated on the pathology request form. Two thirds of respondents thought that the assessment of adequacy is dependent on the menopausal status of the patient. Again, this is not always known to the pathologist because the age at menopause may vary widely. In addition, it might be expected that the use of exogenous hormones and medications, such as tamoxifen, and the type, dose, and duration of usage could influence the amount of tissue obtained at endometrial biopsy. Over half of the respondents stated that there should be correlation with the ultrasonic and/or hysteroscopic findings. However, these investigations are not always performed when obtaining an outpatient endometrial biopsy, and when undertaken the results are not always conveyed to the pathologist.

As can be seen from table 1, the amount of endometrial tissue required by pathologists to categorise an endometrial biopsy as adequate varies widely from any endometrial tissue to a specific number of endometrial glands and stroma in intact organised tissue.

Most respondents felt that it would be useful if criteria for adequacy/inadequacy were to be proposed. Clearly, any such criteria will be arbitrary if not accompanied by published evidence. However, similar criteria have been introduced in other fields of pathology. For example, in breast and thyroid fine needle aspirate specimens, five or six groups of epithelial cells are required to categorise a specimen as adequate. Many respondents to the questionnaire used terms other than inadequate or insufficient when reporting a scanty endometrial biopsy (fig 1). We use the term unassessable.

In summary, our audit and the questionnaire results reveal that there is wide variation among both general pathologists and specialist gynaecological pathologists in the assessment of adequacy of an endometrial biopsy. Although it is difficult to recommend precise criteria for adequacy, we suggest that caution should be exercised before categorising an endometrial biopsy as inadequate or insufficient. In most cases, the presence of only scanty tissue in an endometrial biopsy is not a reason for a repeat biopsy provided the endometrial cavity has been entered. In such cases, the gynaecologist should correlate the biopsy results with the ultrasonic and/or hysteroscopic findings. If there is a clinical suspicion of hyperplasia or malignancy (for example, if there is recurrent postmenopausal bleeding), or if the ultrasonic and/or hysteroscopic findings are worrying, then full dilatation and curettage should be performed. If the above investigations suggest an atrophic endometrium, rebiopsy is unnecessary. Alternative phrases to inadequate or insufficient may be of value, and close liaison between the pathologist and gynaecologist is crucial for optimal patient management.

\section{ACKNOWLEDGEMENTS}

We would like to thank those members of the British Association of Gynaecological Pathologists and National Gynaecological Pathology EQA Scheme who took the time to complete and return the questionnaire. We would also like to thank Mrs M Hornby (administrative secretary of the National Gynaecological Pathology EQA Scheme) for kindly distributing the questionnaires.

\section{Authors' affiliations}

V Phillips, W G McCluggage, Department of Pathology, Royal Group of Hospitals Trust, Grosvenor Road, Belfast BT1 2 6BL, Northern Ireland, UK

Correspondence to: Dr W G McCluggage, Department of Pathology, Royal Group of Hospitals Trust, Grosvenor Road, Belfast BT12 6BL, Northern Ireland, UK; glenn.mccluggage@bll.n-i.nhs.uk

Accepted for publication 21 October 2004

\section{REFERENCES}

1 Clark TJ, Mann CH, Shah N, et al. Accuracy of outpatient endometrial biopsy in the diagnosis of endometrial hyperplasia. Acta Obstet Gynecol Scand 2001;80:784-93.

2 Machado F, Moreno J, Carazo M, et al. Accuracy of endometrial biopsy with the Cornier pipelle for diagnosis of endometrial cancer and atypical hyperplasia. Eur J Gynaecol Oncol 2003;24:279-81.

3 Archer DF, McIntyre-Seltman K, Wilborn WW Jr, et al. Endometrial morphology in asymptomatic postmenopausal women. Am J Obstet Gynecol $1991 ; 165: 317-20$

4 Gordon SJ, Westgate J. The incidence and management of failed pipelle sampling in a general outpatient clinic. Aust N Z J Obstet Gynaecol 1999;39:115-18.

5 Antoni J, Folch E, Costa J, et al. Comparison of cystopat and pipelle endometrial biopsy instruments. Eur J Obstet Gynecol Reprod Biol 1997;72:57-61

6 Stovall TG, Ling FW, Morgan PL. A prospective, randomized comparison of the pipelle endometrial sampling device with the Novak curette. Am J Obstet Gynecol 1991;165:1287-9.

7 Farrell T, Jones N, Owen P, et al. The significance of an "insufficient" pipelle sample in the investigation of post-menopausal bleeding. Acta Obstet Gynecol Scand 1999;78:810-12.

8 Goldstein SR, Nachtigall M, Snyder JR, et al. Endometrial assessment by vaginal ultrasonography before endometrial sampling in patients with postmenopausal bleeding. Am J Obstet Gynecol 1990;163:119-23.

9 Bakour SH, Khan KS, Gupta JK. Controlled analysis of factors associated with insufficient sample on outpatient endometrial biopsy. Br J Obstet Gynaecol 2000;107:1312-14. 